\title{
A Medicina de Família e Comunidade e sua Entidade Nacional: Histórico e PeRspectivas
}

\author{
João Werner Falk *
}

\begin{abstract}
Resumo
O presente trabalho busca relatar a história da Medicina de Família e Comunidade (ex-Medicina Geral Comunitária) no Brasil, bem como da sua entidade nacional representativa, sua interação com o Programa de Saúde da Família e o estabelecimento de propostas para o futuro.
\end{abstract}

Palavras chave

Medicina de Família e Comunidade, Saúde da Família, Atenção Primária em Saúde, História, Brasil. Key Words

\section{Family Practice, Family Health, Primary Care, History, Brazil.}

\section{Introdução}

A Sociedade Brasileira de Medicina de Família e Comunidade (SBMFC) é a entidade nacional que congrega os médicos que atuam em unidades e outros serviços de Atenção Primária em Saúde, incluindo os do Programa de Saúde da Família (PSF), prestando atendimento médico geral, integral e de qualidade a indivíduos, famílias e comunidades. Inclui também professores, preceptores, pesquisadores e outros profissionais que atuam ou estão interessados nesta área.

A SBMFC associa tanto médicos especialistas em Medicina de Família e Comunidade (cujo nome oficial da especialidade e de seus Programas de Residência foi Medicina Geral Comunitária até abril de 2002), quanto outros médicos, residentes e estudantes de medicina interessados nesta área.

Fundada em 1981, nesses seus 20 anos de existência a SBMFC já promoveu diversos eventos nacionais e internacionais, incluindo 5 Congressos Brasileiros de Medicina de Família e Comunidade (antes MGC), tendo o último sido em Curitiba, em 2001. O próximo será no Rio de Janeiro (RJ), de 3 a 6 de abril de 2004, onde também ocorrerá a primeira prova para obtenção de Títulos de Especialista em Medicina de Família e Comunidade.

\section{História}

Para melhor entender o presente e planejar o futuro, é importante conhecer um pouco do passado, e buscar o aprendizado com as experiências, erros e acertos ocorridos. Usando uma comparação aplicada a Medicina de Família e Comunidade (MFC), pode se dizer que "ninguém 'inventou a roda' nos últimos milênios, mas muitos a aprimoraram". Exemplificando, podemos citar o Programa de Saúde da Família (PSF) e outros projetos surgidos na década de 90 que expandiram e, em parte, aperfeiçoaram experiências anteriores. Infelizmente, o PSF até hoje deixa de aproveitar muito da experiência acumulada em quase 30 anos desta área no Brasil.

De modo geral, seria possível dividir a história da MFC no Brasil, como antes e após a implantação do PSF, pelo Ministério da Saúde em 1995. Mas nos parece mais precisa a separação em cinco fases:

\section{Primeira fase - 1974 a 1980}

Em 1974 foi publicado o Projeto de um Sistema de Saúde Comunitária, no Centro de Saúde Murialdo, em Porto Alegre - RS, que parece ser o primeiro projeto e documento conceitual sobre Saúde Comunitária (atualmente reformulada e mais conhecida como Saúde da Família) no Brasil.

Nesta primeira fase, a especialidade e a residência médica na mesma ainda não tinham nome definido e nenhum respaldo normativo ou legal. Existiam poucos Programas de Residência Médica (PRM) no país, incluindo os três pioneiros, iniciados em 1976:

a) Centro de Saúde Escola Murialdo (Porto Alegre - RS) - que, na época, se denominava Unidade Sanitária São José do Murialdo, e que continua ativo até hoje, tendo mais de 600 médicos formados;

b) Projeto Vitória (Vitória de Santo Antão, próximo a Recife - PE), extinto em 1986;

c) Serviço de Medicina Integral da UERJ (Rio de Janeiro - RJ) - que continua ativo e com muitos egressos.

Foi uma "fase experimental" da área e o único, e raro, mercado de trabalho era nos próprios serviços que mantinham os PRM, fazendo com que a maioria dos egressos das residências mudassem de especialidade, a maioria migrando para uma área "afim": a saúde pública. 
Em 1978 houve o maior marco desta área no mundo até hoje - a Conferência Internacional de Cuidados Primários em Saúde, na cidade de Alma Ata / URSS - que reforça o que esses e outros programas no Brasil já vinham fazendo e expande para o mundo a estratégia da Atenção Primária em Saúde (APS).

Daí em diante, foram surgindo no Brasil muitas experiências em APS, localizadas e não articuladas entre si, incluindo vários PRM na área: Petrópolis - RJ, Vitória - ES, Natal - RN, Grupo Hospitalar Conceição - Porto Alegre - RS, Cotia SP, Teresina - PI, São Luiz - MA, Pelotas - RS, Sete Lagoas - MG, Joinville - SC, entre outros. Nem todos sobreviveram aos boicotes do INAMPS, por volta de 1986, e a outros problemas.

Em 1979 foi criada a primeira Residência Multiprofissional em Saúde Comunitária, no Centro de Saúde Murialdo (RS). Segue existindo e tem muitos egressos, entre enfermeiros, odontólogos, psicólogos, assistentes sociais, nutricionistas e outros. Hoje se denomina "Residência Integrada em Saúde" e é regulamentada por Portaria e Lei Estaduais no Rio Grande do Sul.

\section{Segunda fase - 1981 a 1985}

Essa fase inicia com a Resolução 07/81 da Comissão Nacional de Residência Médica (CNRM), em 1981, que formaliza os PRM em Medicina Geral Comunitária (MGC), oficializando esta área como uma especialidade no Brasil, com este nome.

Em novembro do mesmo ano, um grupo de coordenadores de PRMs e fundadores da especialidade no Brasil (psiquiatras, sanitaristas, clínicos, infectologistas, etc.) fundam a Sociedade Brasileira de Medicina Geral Comunitária (SBMGC), com sede em Petrópolis - RJ. Mas essa entidade permaneceu praticamente inativa até 1985, quando, no "I Encontro de Residentes e Ex-Residentes de MGC", em Petrópolis - RJ (primeiro evento nacional da área) se decidiu que os médicos formados em residências na área deveriam reativar a SBMFC e se candidatarem a assumir sua Diretoria.

A especialidade MGC continuava pouco reconhecida, isolada em poucos $P R M s$ no país e quase sem mercado de trabalho.

\section{Terceira fase - 1986 a 1994}

Durante a $8^{\text {a }}$ Conferência Nacional de Saúde, em março de 1986, em Brasília, é reativada a SBMGC, sendo eleita nova Diretoria, só com médicos gerais comunitários, passando a sede para Porto Alegre - RS.

No mesmo ano é fundada a Sociedade Gaúcha de Medicina Geral Comunitária, e outras "Sociedades Estaduais" são organizadas, mas não registradas.
Em setembro de 1986 a SBMGC promove 0 "10 Congresso Brasileiro de Medicina Geral Comunitária" na cidade de Sete Lagoas - MG, onde há intenso e polêmico debate sobre os aspectos conceituais e o nome da especialidade, necessidade de reconhecimento da MGC pelo Conselho Federal de Medicina (CFM) e Associação Médica Brasileira (AMB), o perfil do profissional e sua formação, mercado de trabalho, necessidade de implantação de políticas públicas para à APS, entre outros assuntos. Foram convidados palestrantes de diversos matizes conceituais e ideológicos, incluindo os a favor e os contra essa especialidade.

No mesmo ano é criada a Revista Brasileira de Medicina Geral Comunitária, com um só número publicado, sendo desativada no ano seguinte.

Em outubro de 1986 o Conselho Federal de Medicina (CFM) reconhece a Medicina Geral Comunitária como Especialidade Médica e a Sociedade Brasileira de Medicina Geral Comunitária como sua representante, através da Resolução CFM nำ1.232/86.

Dando seqüência às atividades científicas e de debates intensos, a SBMGC promove o $2^{\circ}$ e o $3^{\circ}$ Congresso Brasileiro de Medicina Geral Comunitária (Florianópolis - SC, em 1997, e Ouro Preto - MG, em 1998).

Nesta fase, intensificam-se as críticas à MGC, tanto da "corporação médica" e da "direita" (considerando a área como "medicina de comunista", estatizante) quanto da Associação Brasileira de Pós-Graduação em Saúde Coletiva (ABRASCO), dos sanitaristas e da "esquerda" (entendendo a MGC como a "Medicina de Família americana disfarçada" da época - modelo "saúde pobre para gente pobre" e "tampão social").

Em 1997, o Instituto Nacional de Assistência Médica da Previdência Social (INAMPS), cuja presidência da época era ligada à ABRASCO, corta todas as bolsas de Residência Médica para Medicina Geral Comunitária no Brasil. A maioria dos Programas são extintos, outros mudam de nome para Medicina Preventiva e Social para não fecharem, poucos sobrevivem.

Em 1988 a diretoria da SBMGC sai de Porto Alegre e vai para Belo Horizonte - MG, mas fica com dificuldades operacionais e acaba desativando essa Sociedade pouco depois.

No mesmo ano a Constituição "Cidadã" é promulgada, estabelecendo a saúde como "direito de todos e dever do Estado".

Em 1990 é criado o Sistema Único de Saúde (SUS), pelas Leis 8.080 e 8.142, gerando condições para implantar diretrizes como descentralização, municipalização, distritalização, participação popular e apoio ao desenvolvimento da Atenção Primária em Saúde (APS).

Neste ano também ocorre a segunda reativação da SBMGC, por iniciativa da Sociedade Gaúcha de MGC, voltando para Porto Alegre a Diretoria da SBMGC. 
Em 1991 a SBMFC promove o 4ํㅡㄹ Congresso Brasileiro de Medicina Geral Comunitária / 10 Congresso Brasileiro Multiprofissional em Saúde Comunitária, em Porto Alegre - RS, sendo um sucesso em número de participantes e no nível dos debates.

No ano seguinte ocorre o $1^{\circ}$ Seminário da "Action in International Medicine" (AIM) na América Latina, em conjunto com a Sociedade Brasileira de Medicina Geral Comunitária, em Porto Alegre.

Em 1994, o Ministério da Saúde - gestão Henrique Santillo, Governo Itamar Franco - cria o Programa de Saúde da Família (PSF), que só veio a crescer de fato vários anos depois. $O$ então Presidente da SBMGC é o primeiro a assinar o documento técnico que estabelece o que é o PSF.

Ainda em 1994, provavelmente em conseqüência da falta de motivação e de mercado de trabalho para a MGC no Brasil, a SBMFC é novamente desativada, por não haver pessoas dispostas a assumirem sua próxima Diretoria.

\section{Quarta fase - 1995 a 2000}

Nesta fase, a partir do PSF e de outras iniciativas, as áreas voltadas à Atenção Básica em Saúde se expandem muito. Numa velocidade bem maior que a capacidade de formação de pessoal. $O$ mercado de trabalho para a MGC começa a crescer, mas eram poucos os médicos especialistas na área, e a qualidade da formação quase sempre deixava a desejar. Buscando minimizar esses problemas, o Ministério da Saúde e outros órgãos criam especializações, cursos de curta duração, Pólos de Capacitação, entre outros, nem sempre com a qualidade desejada. São incrementados estímulos a mudanças no ensino de graduação nas áreas de saúde.

Em outubro de 2000, com o apoio de entidades de Portugal, ocorre o "10 Encontro Luso-Brasileiro de Medicina Geral, Familiar e Comunitária" no Rio de Janeiro - RJ. Na assembléia final foi aprovada a proposta de reaglutinação da categoria, através da reativação da SBMGC. Também ficou decidido que deveria ser intensificado o debate já iniciado sobre a conveniência da mudança de nome da especialidade.

\section{Quinta fase - 2001 até o momento (janeiro de 2004)}

Em março de 2001 a SBMFC é reativada pela terceira vez em sua história e sua nova diretoria é eleita, com membros do RS, RJ e PR e sede em Porto Alegre.

Em agosto do mesmo ano, após longos debates em eventos e pela Internet, seguido de votação em dois turnos pela Internet, decide-se mudar o nome desta Especialidade Médica para "Medicina de Família e Comunidade". A Sociedade Brasileira da área, bem como as suas Sociedades Estaduais filiadas, também mudam para esse nome e atualizam estatutos.

Ainda neste ano, o Ministério da Saúde implanta - Programa de Interiorização do Trabalho em Saúde - PITS, que propõe Especialização Multiprofissional em Saúde da Família, através de treinamento em serviço e ensino à distância, coordenado pelos Núcleos / Pólos de Capacitação do PACS / PSF. A SBMFC e outras instâncias fazem críticas a este Programa, especialmente ao anúncio de concessão de títulos de especialista em um ano e, muitas vezes, sem uma formação adequada e suficiente aos profissionais.

Em novembro de 2001 a SBMFC promove o "5을 Congresso Brasileiro de Medicina de Família e Comunidade", em Curitiba - PR, sendo também um evento multiprofissional em Saúde da Família e tendo grande participação quali e quantitativa.

Em 2002 a Sociedade Brasileira de Medicina de Família e Comunidade (SBMFC) se filia:

a) $\mathrm{Na}$ Confederação Ibero-americana de Medicina Familiar (CIMF).

b) $\mathrm{Na}$ Organização Mundial dos Médicos de Família (WONCA).

c) $\mathrm{Na}$ Associação Médica Brasileira (AMB) filiação aceita, mas que só foi homologada em novembro de 2003.

Nesta quinta e atual fase, o PSF mais que triplica sua dimensão, passando de 20.000 equipes no país, intensifica-se 0 descompasso entre a enorme ampliação do mercado de trabalho e a pequena escala de formação e capacitação de profissionais em APS - além do que, muitas vezes com deficiências de qualidade e carência de acreditação ou de reconhecimento formal de vários desses programas de desenvolvimento de pessoal para a área.

Esse período é marcado também pela expansão e consolidação (acreditamos que agora definitiva) da SBMFC, que cresceu muito em número de sócios em todo o país, passou a ter contatos freqüentes e a conquistar influência saudável e ativa junto a órgãos ou entidades como o CFM, a Comissão Nacional de Residência Médica (CNRM), a AMB, a Federação Nacional dos Médicos (FENAM), a Organização Panamericana de Saúde (OPAS), a WONCA, a CIMF e ao Ministério da Saúde - neste, principalmente na gestão que iniciou em 2003 - Governo Luiz Inácio Lula da Silva.

Em 2003 a SBMFC chegou à sua $10^{\text {a }}$ Sociedade Estadual - na ordem de filiação: RS, PR, SC, RJ, $\mathrm{SP}, \mathrm{MG}, \mathrm{DF}, \mathrm{CE}, \mathrm{AM}$ e GO. Outras estão em fase de criação.

Em novembro de 2003 a SBMFC conquistou sua filiação definitiva à $A M B$, que havia sido novamente solicitada abril de 2001. A decisão final foi do Conselho Científico da AMB, vencendo por vinte e dois votos a zero outra entidade que havia também se candidatado à filiação meses 
antes, e provocado mais um adiamento da homologação da filiação da SBMFC.

Em dezembro de 2003 a AMB aprovou os dois Editais de Concurso para Título de Especialista em Medicina de Família e Comunidade (TEMFC) elaborados pela SBMFC, autorizando-a a publicálos.

O Edital 1 para o TEMFC é voltado para titular um percentual muito pequeno de médicos, que comprovem ser os mais capacitados na especialidade, para que destes sejam escolhidas as bancas de avaliação para o Edital 2, que inclui análise de currículo e uma prova escrita que ocorrerá no dia 3 de abril de 2003 , no Rio de Janeiro, logo antes da abertura do "6으 Congresso Brasileiro de Medicina de Família e Comunidade, e Congresso Mercosul da CIMF", na mesma cidade. A expectativa é de um grande número de inscritos tanto para o Edital 2 quanto para o Congresso.

\section{Propostas da SBMFC para o Futuro}

A SBMFC trabalha pelo fortalecimento do movimento dos profissionais que atuam em Atenção Primária em Saúde (APS), incluindo os do Programa de Saúde da Família (PSF). Para tanto, no caso dos médicos, está buscando:

Integrar na SBMFC os médicos da área em todo - Brasil, com uma entidade filiada em cada estado (algumas existentes e outras a serem criadas) e ampliar o número de associados e a participação dos mesmos.

Colaborar com o Ministério da Saúde, Secretarias Estaduais e Municipais de Saúde e outros órgãos na tarefa de desenvolver, aprimorar e fortalecer politicamente a Atenção Primária em Saúde, a Medicina de Família e Comunidade, a Saúde da Familia e áreas afins, como um todo integrado no Brasil, com qualidade.

Favorecer a qualificação, a atualização e educação continuada do médico que oferece atenção integral e continuada de primeira linha às populações.

Estimular sistema de acreditação de Programas de Residência, Especialização e outros nestas áreas.

Apoiar, criteriosamente, programas de especialização em Saúde Comunitária / Saúde da Familia para múltiplas profissões de saúde, em ambiente de convívio interdisciplinar, com treinamento em serviço (incluindo a possibilidade do formato de Residência Integrada em Saúde) com acreditação, para terem qualidade.

Mesmo reconhecendo a primazia da Residência Médica, propor um plano nacional de especialização e reciclagem em MFC, bem como articulá-lo com a concessão de Títulos de Especialista.

Conceder, criteriosamente, Títulos de Especialista em Medicina de Família e Comunidade pela SBMFC - o primeiro concurso de provas e currículo ocorrerá em abril de 2004, na véspera do 6ํㅡㄹ Congresso Brasileiro de MFC.

Realizar ou colaborar na acreditação de serviços de APS ou Atenção Básica em saúde (incluindo os do PSF).

Estimular que as equipes do PSF e demais serviços de APS atuem com profissionais com perfil adequado e realmente capacitado para este trabalho. No caso dos médicos, que priorizem os especialistas em Medicina de Família e Comunidade (por Residência ou por Título de Especialista concedido pela SBMFC).

Colaborar com a elaboração de um Plano de Carreira Nacional (ou diretrizes nacionais para Planos de Carreira Municipais, se for o caso).

Lutar por direitos trabalhistas e condições de trabalho dignas, através do estabelecimento de vínculos empregatícios legais e éticos e outras situações que qualifiquem o exercício profissional da especialidade.

Desenvolver a produção científica e sua divulgação, incluindo o lançamento da Revista Brasileira de Medicina de Família e Comunidade.

Criar linhas de pesquisa, mestrados e doutorados em MFC.

Buscar o desenvolvimento da MFC e da APS no Brasil, de forma consciente, crítica e autônoma, prestando, assim, sua parcela de colaboração para a melhoria das condições de vida e saúde da população brasileira.

\section{Bibliografia Consultada}

ASSOCIAÇÃO MÉDICA BRASILEIRA. Decisão do Conselho Científico. Filia a Sociedade Brasileira de Medicina de Família e Comunidade (SBMFC) e a responsabiliza por representar a especialidade Medicina de Família e Comunidade junto à AMB. São Paulo, 25 de novembro de 2003.

ASSOCIAÇÃO MÉDICA BRASILEIRA. Aprova os Editais para Concursos para Títulos de Especialista em Medicina de Família e Comunidade pela SBMFC. São Paulo, 30 de dezembro de 2003 ASSOCIAÇÃO NACIONAL DOS MÉDICOS RESIDENTES Legislação da Residência Médica. Belo Horizonte, Cadernos da ANMR, n.1, 1987.

ASSOCIAÇÃO NACIONAL DOS MÉDICOS RESIDENTES. Pesquisa sobre a Residência Médica: histórico do movimento. Belo Horizonte, Cadernos da ANMR, n.2, 1987

BORDIN, Ronaldo \& SILVA, Jacqueline O. Análise Crítica dos Programas de Residência Médica em Medicina Geral Comunitária. Revista HCPA, Porto Alegre, v.9, n.1, abril de 1989. p.34:39.

BORDIN, Ronaldo \& SILVA, Jacqueline O. Medicina Geral Comunitária: Novos Rumos? Rev. Bras. Educação Médica, Rio de Janeiro, v.12, n.1, p.1-40, jan/abr. 1988.

BRASIL - MINISTÉRIO DA EDUCAÇÃO - SESu - CNRM. Resolução CNRM n 07/81: Inclui o Programa de Residência Médica em Medicina Geral Comunitária entre as Especialidades Médicas credenciáveis pela CNRM. Brasília, Diário Oficial da União de 17 de junho de 1981, página 11.436.

BRASIL - MINISTÉRIO DA EDUCAÇÃO - SESU - CNRM. Resolução CNRM nº 16/81: Dispõe sobre normas de Residência Médica em Medicina Preventiva e Social, revoga a Resolução CNRM n 08/79 e dá outras providências. Brasília, Diário Oficial da União de 22 de outubro de 1981.

BRASIL - MINISTÉRIO DA EDUCAÇÃO - SESu - CNRM Resolução CNRM n $\mathrm{n}^{\circ}$ 05/2002: Atualiza as especialidades credenciáveis pela CNRM e as exigências para cada uma, incluindo a Medicina de Família e Comunidade, já com esse novo nome. Brasília, dezembro de 2002. Republicado, com correções no Diário Oficial da União de 23 de dezembro de 2003. 
BUSNELLO, Ellis A.D. \& LEWIN, Isaac. O Tipo de Médico que as Escolas de Medicina Devem Graduar. Rev. CASL. Porto Alegre, Centro Acadêmico Sarmento Leite da Faculdade de Medicina da UFRGS, n.34, p.11-27, 1973

BUSNELLO, Ellis A.D. A Integração da Saúde Mental num Sistema de Saúde Comunitária. Porto Alegre, 1976. (Tese destinada à habilitação a Livre-Docência em Psiquiatria junto ao Departamento de Psiquiatria e Medicina Legal da Faculdade de Medicina da UFRGS).

BUSNELLO, Ellis A.D. et al. O Projeto do Sistema de Saúde Comunitária. Centro Médico Social São José do Murialdo da Secretaria da Saúde do Rio Grande do Sul. Porto Alegre, out. 1974/abr. 1975.

CARRICONDE, Celerino. Medicina Comunitária: Uma Concepção e um Método de Trabalho. $2^{\text {a }}$ Ed., Petrópolis, Vozes, 1986. CARTA DE OTTAWA. Ottawa, 21 de novembro de 1986.

CARTA DO CARIBE. Port of Spain, Trinidad y Tobago, 4 de junho de 1993.

CARTA DE FORTALEZA. Seminário Internacional Sobre Saúde da Família: formação, certificação e educação permanente do médico de família. Fortaleza, abril de 2001.

CHAVES, Paulo O. (coord.) Avaliação do Sistema do Murialdo. Porto Alegre, 1983. 217 p. (pesquisa realizada pela Assessoria de Planejamento da Secretaria da Saúde e do Meio Ambiente do Estado do Rio Grande do Sul, onde estão arquivados exemplares).

CONILL, Eleonor M. Contribuição ao Desenvolvimento da Medicina Geral Comunitária nos Sistemas Estaduais de Saúde. Rio de Janeiro, 1986. (mimeo)

CONSELHO FEDERAL DE MEDICINA. Processo Consulta CFM 29/86. Brasília, 11 de junho de 1986. (em www.cremesp.org.br/legislacao/pareceres/parcfm/29_1986.htm ) CONSELHO FEDERAL DE MEDICINA. Resolução CFM $n^{\circ}$ 1.232/86: Reconhece a Medicina Geral Comunitária como Especialidade Médica para efeito de Registro de Qualificação de Especialistas nos Conselhos de Medicina. Diário Oficial da União de 23 de outubro de 1986, página 15.949 .

CONSELHO FEDERAL DE MEDICINA. Convênio Celebrado com a Associação Médica Brasileira para Regulamentar a Concessão e Registro de Títulos de Especialista. Brasília, 10 de março de 1989. CONSELHO FEDERAL DE MEDICINA. RESOLUÇÃO CFM N $1.666 / 2003$. Dispõe sobre a nova redação do Anexo II da Resolução CFM n ${ }^{\circ} 1.634 / 2002$, que celebra o convênio de reconhecimento de especialidades médicas firmado entre o Conselho Federal de Medicina - CFM a Associação Médica Brasileira - AMB e Comissão Nacional de Residência Médica - CNRM. Brasília, 7 de maio de 2003

CUBA - MINISTERIO DE SALUD PUBLICA. ProgramaResumenes del "II Seminario Internacional de Atencion Primaria de la Salud: El Medico de la Familia, una Respuesta a las Necessidades de la Comunidad". Havana, 14 a 18 de novembro de 1988.

DECLARAÇÃO DE EDIMBURGO (sobre Educação Médica). Edimburgo, 12 de agosto de 1988

ELIAS, Paulo E. M. Regulamentação da Residência Médica: entre as técnicas pedagógicas e as Políticas de Saúde. São Paulo, 1986 (documento apresentado no II Fórum Nacional sobre Residência Médica, em novembro de 1986, em Brasília).

ELIAS, Paulo E. M. Residência Médica no Brasil: a institucionalização da ambivalência. São Paulo, 1987 (Dissertação de Mestrado apresentada na Faculdade de Medicina da USP)

FACULDADE DE MEDICINA DE PETRÓPOLIS. Relatório Final do I Encontro Nacional de Médicos Residentes e Ex-Residentes de Medicina Geral Comunitária. Petrópolis - RJ, novembro de 1985.

FALK, João W. Residência Médica no Brasil: Especialização, Emprego ou Reprodução Ideológica? Porto Alegre, julho de 1990 (texto apresentado à Disciplina "Teoria e Prática do Planejamento da Educação" no Programa de Pós-graduação em Educação da UFRGS).

FALK, João W. Aspectos Conceituais, Históricos e Curriculares da Medicina Geral Comunitária. In: Pedagogia Universitária: conhecimento, ética e política no ensino superior. Porto Alegre: Editora da Universidade/UFRGS, 1999

FALK, João W. Breve Histórico da Medicina de Família e Comunidade no Brasil. Porto Alegre, 2001 (atualizado em 2003). Disponível na página da SBMFC na Internet, em www.sbmfc.org.br FUNDAÇÃO OSVALDO CRUZ \& CONSELHO FEDERAL DE MEDICINA. Perfil dos Médicos no Brasil. 1996.
JORNAL DO SIMERS. Médicos Gerais Comunitários Reivindicam Reconhecimento da Especialidade. Sindicato Médico do Rio Grande do Sul, Porto Alegre, janeiro de 1986.

JORNAL ZERO HORA. Médicos Generalistas Exigem Reconhecimento. Porto Alegre, 09 de dezembro de 1985.

JORNAL ZERO HORA. A Medicina Comunitária em Debate. Porto Alegre, 06 de março de 1986.

JORNAL ZERO HORA. Congresso Pede Maior Espaço Para os Médicos Gerais Comunitários. Porto Alegre, 08 de março de 1986.

JORNAL ZERO HORA. Propostas de Ação do Médico Geral Comunitário. Porto Alegre, 09 de março de 1986.

LEITE, Denise B.C. Metacognição e Aprendizagem na Universidade. Revista de Educação do Departamento de Educação da F.C. da Universidade de Lisboa, v.2, n.2, out. 1992.

LEME, Luiz E.G. Medicina de Família, Solução Moderna de Antigamente. Consultário Médico, São Paulo, nov/dez 1992.

LEONARDELLI, Natal \& ROSA, Roger S. Histórico e Planos de Ensino da Residência em Medicina Preventiva e Social / HCPA Revista HCPA, Porto Alegre, v.10, n.1, p.38-47, abril 1990.

MACHADO, Vera L.M. Abordagem Comunitária em Saúde: conceitos, proposições e limites. Município e Saúde. Ed. Unijuí, ano $2 \mathrm{n}^{\circ} 3,1994$

MENDES, Eugênio V. A Evolução Histórica da Prática Médica. Belo Horizonte, 1985.

ORGANIZAÇÃO MUNDIAL DE SAÚDE \& UNICEF. Declaração de Alma-Ata (Conferência Internacional Sobre Cuidados Primários de Saúde). Alma-Ata, URSS, 1976.

ORGANIZAÇÃO MUNDIAL DE SAÚDE. O Trabalhador de Saúde Comunitária. Porto Alegre, agosto de 1979. (Publicação da tradução realizada pela Equipe do Murialdo do livro da OMS "The Primary Health Worker", de 1976).

POZATTI, Mauro L. Paradigmas Médicos e Práticas Médicas: Análise de suas Influências em um Estágio de Medicina Comunitária da UFRGS. Porto Alegre, 1990. (Dissertação de Mestrado apresentada ao Programa de Pós-graduação em Educação da UFRGS).

RIO GRANDE DO SUL - Secretaria da Saúde - Unidade Sanitária Murialdo e Escola de Saúde Pública. Projeto: Residência em Medicina Geral Comunitária / Curso de Especialização em Saúde Comunitária ao Nível de Residência. Porto Alegre, agosto de 1991. RIO GRANDE DO SUL. Secretaria da Saúde. Portaria 1699: cria a Residência Integrada em Saúde - RIS. Porto Alegre, 1999.

RIO GRANDE DO SUL. Lei Estadual 11789/2002. Cria o programa de bolsas para a Residência Integrada em Saúde. Porto Alegre, 2002. SAN MARTIN, H.; PASTOR, V. Salud Comunitaria - teoria y pratica. Madrid, Ed. Diaz de Santos S.A., 1984

SCHRAIBER, Lilia. Educação Médica e Capitalismo: Um Estudo das Relações Educação e Prática Médica na Ordem Socia Capitalista. São Paulo, Hucitec-Abrasco, 1989. 133 p.

SILVA, Lucia S. Municipalização da Saúde: Reflexões Sobre Como e com que Equipe Implementá-la. Contexto \& Educação, Unijuí, n.25, jan/mar. 1992. p.92-98.

SILVA, Nilton B. Qual o Médico de que Necessitamos para a Década de 1990? Revista Médica da Santa Casa. Porto Alegre, v.3, n.5, p.561-562, 1991

SOCIEDADE BRASILEIRA DE MEDICINA DE FAMÍLIA E COMUNIDADE. Relatório Final do V Congresso Brasileiro de Medicina de Família e Comunidade. Curitiba - PR, 9 a 13 de novembro de 2001

SOCIEDADE BRASILEIRA DE MEDICINA GERAL COMUNITÁRIA - SBMGC. Ata de Fundação da SBMGC Petrópolis-RJ, 1981.

SOCIEDADE BRASILEIRA DE MEDICINA GERAL COMUNITÁRIA. Ata $n^{\circ}$ 02/86: Elege a Diretoria da SBMGC recém reativada. Porto Alegre, 16 de abril de 1986

SOCIEDADE BRASILEIRA DE MEDICINA GERAL COMUNITÁRIA. Boletim Informativo $\mathrm{n}^{\circ} 2$, ano I, 8p. Porto Alegre, outubro de 1986

SOCIEDADE BRASILEIRA DE MEDICINA GERAL COMUNITÁRIA. Revista Brasileira de Medicina Gera Comunitária. Porto Alegre, v.1, n. 1, julho 1987.

SOCIEDADE BRASILEIRA DE MEDICINA GERAL COMUNITÁRIA. Ata de alteração de nome para Sociedade Brasileira de Medicina de Família e Comunidade e Aprovação dos Novos Estatutos. Porto Alegre - RS, março de 2001. 
SOCIEDADE BRASILEIRA DE MEDICINA GERAL COMUNITÁRIA. Ata de Reativação da SBMGC. Brasília, 19 de março de 1986.

SOCIEDADE BRASILEIRA DE MEDICINA GERAL COMUNITÁRIA. Relatório Final do I Congresso Brasileiro de Medicina Geral Comunitária. Sete Lagoas-MG. In: Boletim Informativo $\mathrm{n}^{\circ} 2$, ano I, p. 4-5. Porto Alegre, outubro de 1986.

SOCIEDADE BRASILEIRA DE MEDICINA GERAL COMUNITÁRIA. Relatário Final do II Congresso Brasileiro de Medicina Geral Comunitária. Florianópolis - SC, 7 a 11 de setembro de 1987.

SOCIEDADE BRASILEIRA DE MEDICINA GERAL COMUNITÁRIA. Relatório Final do III Congresso Brasileiro de Medicina Geral Comunitária. Ouro Preto - MG, agosto de 1988.

SOCIEDADE BRASILEIRA DE MEDICINA GERAL COMUNITÁRIA. Relatório Final do IV Congresso Brasileiro de Medicina Geral Comunitária e I Congresso Brasileiro Multiprofissional de Saúde Comunitária. Porto Alegre - RS, 9 a 13 de setembro de 1991

SOCIEDADE BRASILEIRA DE MEDICINA GERAL COMUNITÁRIA. Ata de reativação da Sociedade Brasileira de Medicina Geral Comunitária. Porto Alegre - RS, março de 2001.

SOCIEDADE GAÚCHA DE MEDICINA GERAL COMUNITÁRIA. Relatório Final do I Encontro Sul-Brasileiro de Médicos Gerais Comunitários. Porto Alegre, 7 e 8 de março de 1986. TAVARES, Mário; TAKEDA, Sílvia. A Prática da Atenção Primária à Saúde. In: DUNCAN, Bruce B. et al. Medicina Ambulatorial: condutas clínicas em atenção primária. 2.ed. Porto Alegre. Ed. Artes Médicas, 1996.

WONCA. A Definição Européia de Medicina Familiar. Versão para o português da Associação Portuguesa dos Médicos de Clínica Geral. Lisboa, 2002.

* Professor do Departamento de Medicina Social da Faculdade de Medicina da Universidade Federal do Rio Grande do Sul. Presidente da Sociedade Brasileira de Medicina de Família e Comunidade (atualmente e em quatro gestões anteriores, nem sempre consecutivas).

Endereço para correspondência: João Werner Falk.

Rua São Manoel, 120 CEP 90620-110

Porto Alegre, RS

E-mail: falk@orion.ufrgs.br 